RESEARCH NOTES

\title{
SPLAYING A SEARCH TREE IN PREORDER TAKES LINEAR TIME
}

\author{
R. CHAUDHURI and H. HÖFT
}

Department of Computer Science

Eastern Michigan University

Ypsilanti, Mich. 48197.

1. Abstract

(Received September 15,1989)

In this paper we prove that if the nodes of an arbitrary n-node binary search tree $T$ are splayed according to the preorder sequence of $T$ then the total time is $O(n)$. This is a special case of the splay tree traversal conjecture of Sleator and Tarjan [1].

\section{Introduction}

A binary search tree in which we splay after each access to the node containing the accessed item is called a splay tree. Splaying is a restructuring operation consisting of a sequence of rotations (see [1] for details). Tarjan [2] proved that the nodes of an $n$-node search tree can be splayed in symmetric order (inorder) in $O(n)$ time.

We define the splay depth (SD) of a node $x$ to be the depth of $x$ at the time we start splaying at $x$. The set of all the right ancestors of a node $z$ in the subtree rooted at a node $x$ is denoted by $A(z, x)$. Also, left( $x)$ and right $(x)$ denote the left child and the right child of a node $x$ respectively.

\section{Main Results}

In the following, we simply state our main results.

\section{THEOREM 1}

Let $T$ be a binary search tree whose nodes are being splayed according to its own preorder sequence. Let $x$ be a node of $T$ and assume that the splay depth (SD) of $x$ is $d$. Then

(a) $\operatorname{SD}(\operatorname{left}(x)) \leqslant d / 2+3 / 2$ and

(b) $S D($ right $(x)) \leqslant 1+|A(z, x)|$

where $z$ is the preorder predecessor of $\operatorname{right}(x)$ and $|A(z, x)|$ denotes the cardinality of the set $A(z, x)$.

Using Theorem 1, we prove that :

\section{THEOREM 2}

The total time to splay an $n$-node binary search tree $T$ according to its own preorder sequence is at most $8 \mathrm{n}$.

The complete paper is being submitted for publication in the Journal of the Association for Computing Machinery.

\section{References}

1. D.D.Sleator and R.E.Tarjan: "Self-adjusting binary search trees", Journal of the Association for Computing Machinery, 32, 1985, 652-686.

2. R.E.Tarjan: "Sequential access in splay trees takes linear time", Combinatorica, 5(4), 1985, 367-378.

3. R.E.Tarjan: "Data Structures and Network Algorithms", C.B.M.S 44 (1983), S.I.A.M, Philadelphia, $\mathrm{Pa}$. 


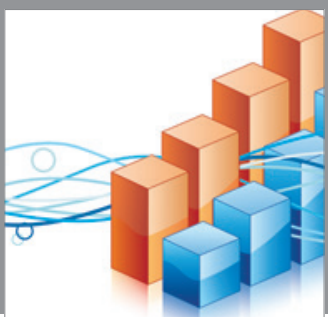

Advances in

Operations Research

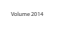

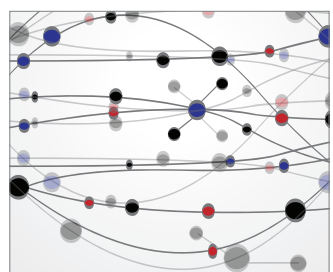

\section{The Scientific} World Journal
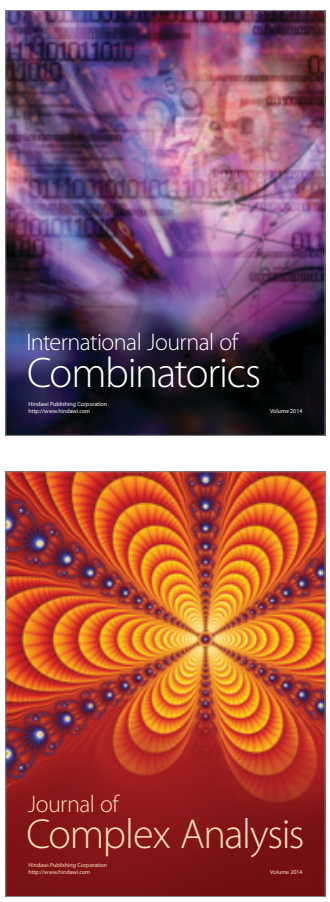

International Journal of

Mathematics and

Mathematical

Sciences
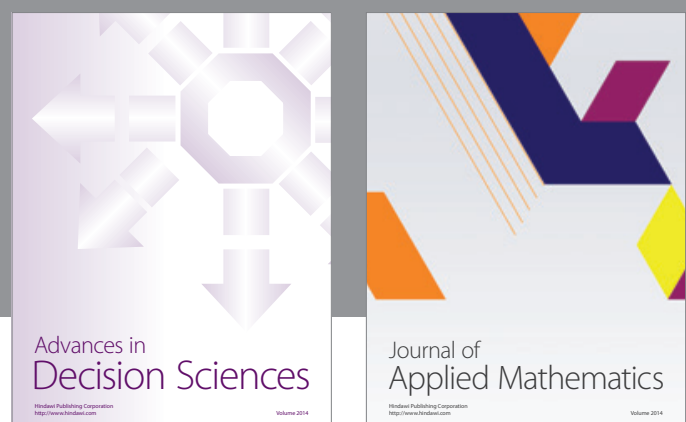

Journal of

Applied Mathematics
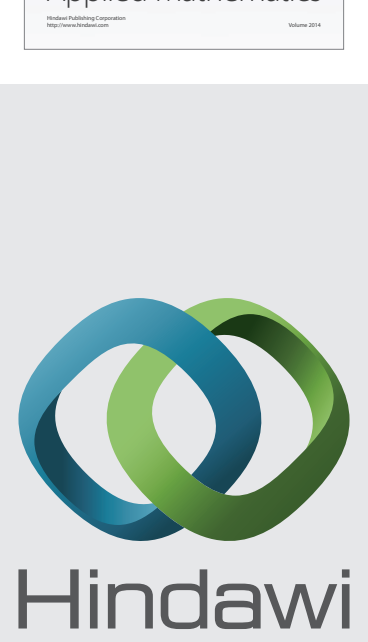

Submit your manuscripts at http://www.hindawi.com
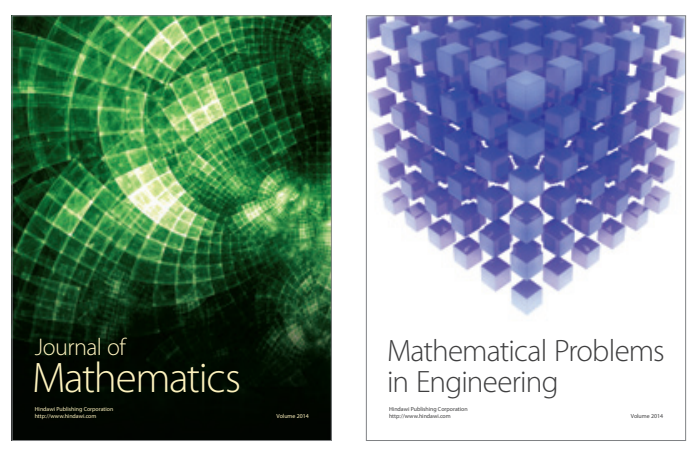

Mathematical Problems in Engineering
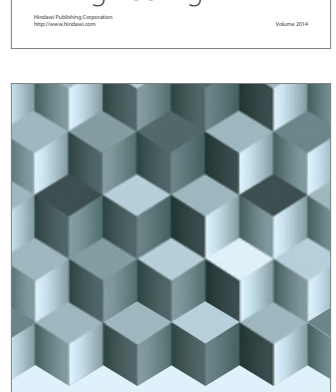

Journal of

Function Spaces
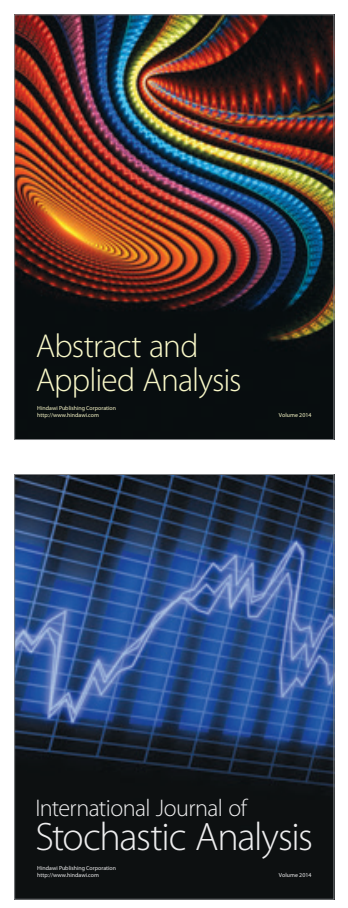

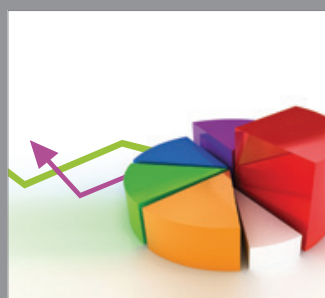

ournal of

Probability and Statistics

Promensencen
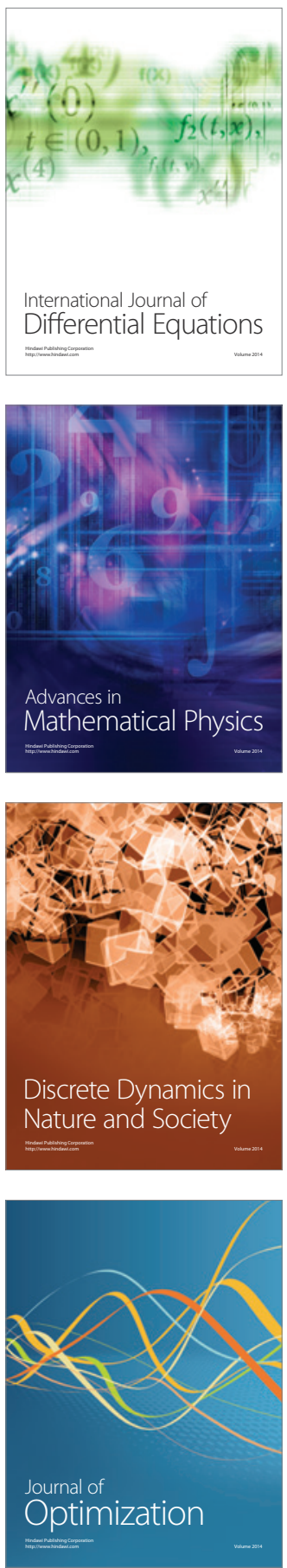